\title{
Fault features analysis for soft faults of analog circuits with tolerance
}

\author{
Chaojie Zhanga , Guo He, Jiankeng Yu, and Xionglong Pan \\ College of Power Engineering, Naval University of Engineering, 717 Jiefang Street, Wuhan, China
}

\begin{abstract}
Soft faults of analog circuits are more difficult to diagnose than hard faults because the soft faults are caused by the deviations of component parameters. Node voltages were traditionally used as the testing signals to diagnose analog circuits. With the rapid development of integrated circuits technology, fewer and fewer circuit nodes are accessible. Only the output voltage can be tested in many cases. This cries for other new accessible signal except for the traditional voltage signal. In this paper, the fault features of testing signal in both time-domain and frequency-domain are analysed. The output voltage was acquired firstly. Its fault features were extracted and used for fault diagnosis. The results show that the soft faults of TowThomas filter cannot be uniquely located by using this output voltage only. Then a new accessible signal, which named dynamic power supply current, was acquired and its fault features were analysed. And the results were compared with those using output voltages. The comparing results show the validity of dynamic power supply current. This signal contains information related with the circuits' topology and can be used for fault diagnosis of analog circuits.
\end{abstract}

\section{Introduction}

The electronic circuits are used widely in many automation systems. These circuits can be classified into two categories: analog circuits and digital circuits. Compared to digital circuits, analog circuits are more difficult to locate faults due to poor fault models, nonlinearity characters, and ubiquitous component tolerances [1]. Analog circuits' diagnosis is the bottleneck of electronic circuits at present. It is an active area of research with significant work carried out at the system, board, and chip level [1-8]

Faults of analog circuits can be classified into two categories: soft faults and hard faults. Soft faults are deviations of component parameters which result in performance out of acceptable limits. Compared with hard faults which happen due to excessive deviations, soft faults are by far more difficult to diagnose because they are caused by the deviations of component parameters [5].

Node voltages are often used as the testing signals to diagnose analog circuits. The voltages of some accessible nodes are acquired. Then the fault features of voltage signals are extracted to find the differences of electronic circuits with different faults. With the rapid development of IC technology, fewer and fewer circuit nodes are accessible. Even only the output voltage signal is accessible in many cases. So, the rapid development of integrated circuits is crying for other new accessible signal except for the traditional voltage signal.

In this paper, the output voltage is used as an accessible signal to diagnose analog circuits firstly. The fault features of output voltage are extracted and analysed. Then, a new accessible signal, which named dynamic power supply current, is used to diagnose the same analog circuits too. The fault features of these two kinds of signals were analysed and compared for fault diagnosis of analog circuits.

The rest of this paper is organized as follows. Fault diagnosis of analog circuits using output voltage are discussed in Section 2. The fault features of output voltage are extracted to build the fault dictionary for fault diagnosis. As an example, a signal filtering circuit is used as the circuits-under-test (CUT). In Section 3, we use the dynamic power supply current to diagnose the same CUT. The fault features of dynamic power supply current are extracted to build the fault dictionary, too. The results of fault diagnosis using these two kinds of signals are compared in Section 4. Section 5 concludes this paper.

\section{Fault features analysis of output voltage}

The Tow-Thomas filter is used as a CUT in this paper, because it is used widely in many kinds of electronic circuits. It is composed with three operational amplifiers, six resisters and two capacitors. It can be used as highpass filter, low-pass filter or band-pass filter. In our research, it is used as a band-pass filter. The nominal values of the components which result in a characteristic frequency of $1 \mathrm{kHz}$ are shown in Figure 1. The soft faults of this filter are considered as the potential faults.

*Corresponding author: ${ }^{\mathrm{a}}$ zcjdoctor@ $126 . c 0 m$ 
Only several components are selected as the potential faults of analog circuits in many researches [1-6]. This is inappro-priate, because they often selected the components which were easy to locate. In our research, the soft faults of all the resisters and capacitors are considered. The potential faults and their corresponding codes are shown in Table 1. In this notation, ' $\uparrow$ ' and ' $\downarrow$ ' stand for high and low, respectively. 'Nor' stands for the fault-free state of circuits. When any of the components is higher or lower than its nominal value by $50 \%$, we think it is faulty.

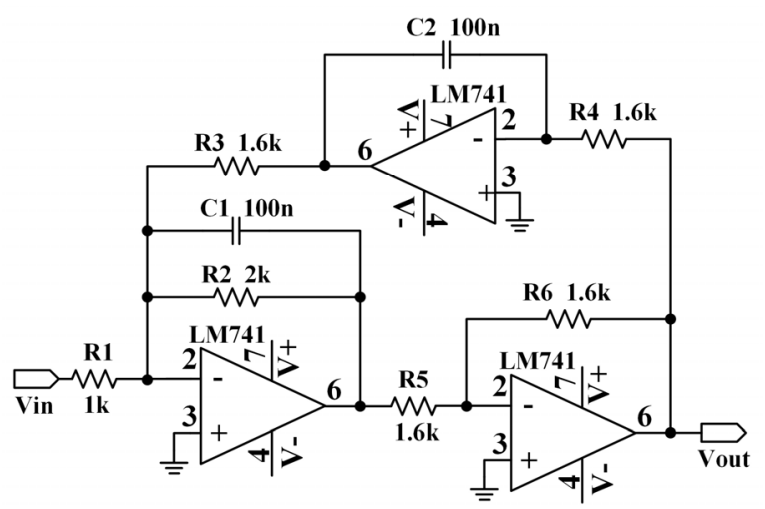

Figure 1. Tow-Thomas filter

Table 1. Faults and their corresponding codes

\begin{tabular}{c|cccccc}
\hline Faults & $\mathrm{R} 1 \uparrow$ & $\mathrm{R} 1 \downarrow$ & $\mathrm{R} 2 \uparrow$ & $\mathrm{R} 2 \downarrow$ & $\mathrm{R} 3 \uparrow$ & $\mathrm{R} 3 \downarrow$ \\
Codes & $f_{1}$ & $f_{2}$ & $f_{3}$ & $f_{4}$ & $f_{5}$ & $f_{6}$ \\
\hline Faults & $\mathrm{R} 4 \uparrow$ & $\mathrm{R} 4 \downarrow$ & $\mathrm{R} 5 \uparrow$ & $\mathrm{R} 5 \downarrow$ & $\mathrm{R} 6 \uparrow$ & $\mathrm{R} 6 \downarrow$ \\
Codes & $f_{7}$ & $f_{8}$ & $f_{9}$ & $f_{10}$ & $f_{11}$ & $f_{12}$ \\
\hline Faults & $\mathrm{C} 1 \uparrow$ & $\mathrm{C} 1 \downarrow$ & $\mathrm{C} 2 \uparrow$ & $\mathrm{C} 2 \downarrow$ & Nor & \\
Codes & $f_{13}$ & $f_{14}$ & $f_{15}$ & $f_{16}$ & $f_{0}$ & \\
\hline
\end{tabular}

The power supplies for this circuit are a $12 \mathrm{~V} \mathrm{DC}$ and a $-12 \mathrm{~V}$ DC. The input stimulus is a $2 \mathrm{~V}(\mathrm{p}-\mathrm{p}), 1 \mathrm{kHz}$ sinusoidal wave. The output voltage generated by such an input stimulus is sampled. To analyse the fault diagnosis effectiveness using output voltages, the waveforms of output voltages are sampled and saved for the fault-free case and all the faulty cases shown in Table 1. For example, Figure 2 shows the waveform of output voltage when the filter has the faulty type $f_{1}$.

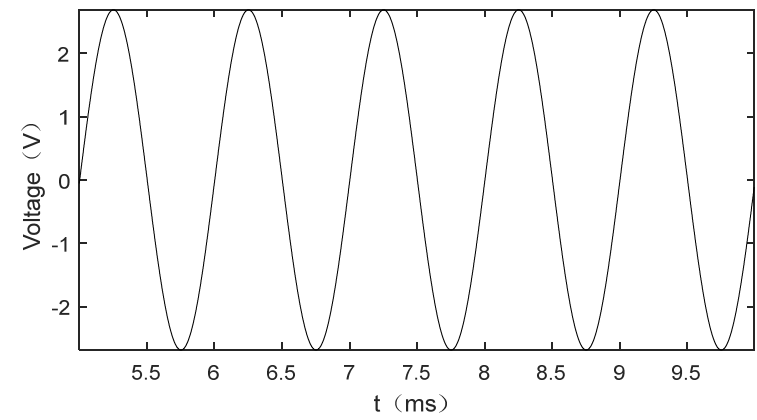

Figure 2. Waveforms of output voltage for the faulty type $f_{1}$

In order to analyse the waveform of output voltage, the features both in time-domain and frequency-domain are extracted. The selected features of time-domain are: the maximum value $t_{1}$, the minimum value $t_{2}$, the mean value $t_{3}$ and the second maxima value $t_{4}$. The features of frequency-domain are the magnitude component values of the first four harmonics, donated as $t_{5}, t_{6}, t_{7}, t_{8}$ in this paper. These eight fault features and the fault types are used to construct the fault dictionary. In the fault dictionary, rows represent all the potential faults (including fault-free state) and the columns represent these eight fault features.

For a fault feature, its value may not be certain because of the components' tolerances. The value often fluctuates in an interval. Therefore, the values of a fault feature for different faults may be the same or their intervals are overlapped. The concept of ambiguity set was first introduced by Hochwald and Bastian [7]. It is defined as a list of faults which have 'similar' fault features (Their fault features cannot be distinguished). A method of integer-coded faulty-wise dictionary was proposed for fault diagnosis of analog circuits [8]. For each fault feature, a specific integer code is assigned to each ambiguity set. The same integer code represents the faults which belong to the same ambiguity set for a given fault feature. However, the ambiguity sets of each fault feature are independent and can be assigned with the same integer code without confusion.

Let $F=\left\{f_{0}, f_{1}, \cdots, f_{16}\right\}$ be the set of all potential faults and $T=\left\{t_{0}, t_{1}, \cdots, t_{8}\right\}$ be the set of fault features. To define the concept of diagnosable circuit, we associate the measurement equivalent classes which are the elements of the dictionary $A$ as follows:

$$
\begin{aligned}
& \forall f_{i} \in F(0 \leq i \leq 16) \quad \text { and } \quad \forall t_{j} \in T(1 \leq j \leq 8) \\
& \exists \phi\left(f_{i}, n_{j}\right)=a_{i j} \in A
\end{aligned}
$$

Where, $a_{\mathrm{ij}}$ is element of dictionary $A$ corresponding to the $i$ th fault and $j$ th fault features.

The ambiguity sets for fault features of output voltage are shown in Figure 3. According these ambiguity sets, the integer-coded faulty-wise dictionary are constructed and shown in Table 2 . The ambiguity sets are the same among the maximum value $t_{1}$, the minimum value $t_{2}$, and the first magnitude component value $t_{5}$. So, we can use one of these three fault features. The mean values $t_{3}$ of output voltages for all the faulty cases are equal to zero. it only has one ambiguity set. So, the mean value $t_{3}$ cannot distinguish any faults. The second maxima value $t_{4}$ only has one ambiguity set, too. The ambiguity sets are the same between the third magnitude component value $t_{7}$ and the fourth magnitude component value $t_{8}$. So, we can use one of them. According these analysis, the integer-coded faulty-wise dictionary of Table 2 can be simplified as follows: removing the columns which corresponding to $t_{2}, t_{3}, t_{4}, t_{5}$, $t_{8}$. 


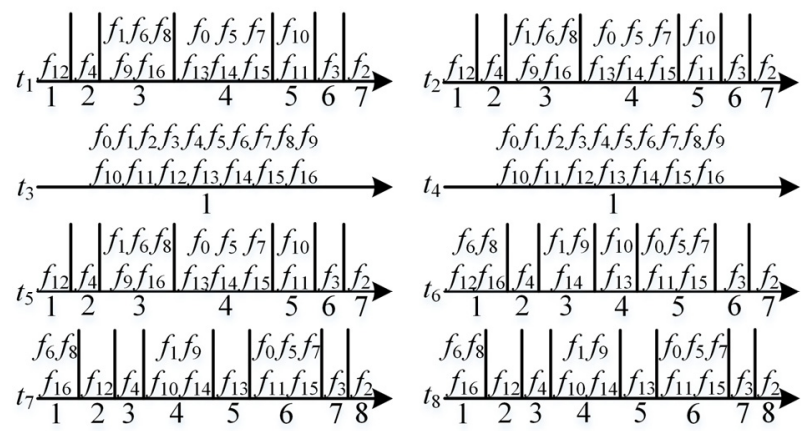

Figure 3. Ambiguity sets for fault features of output voltage

This simplified fault dictionary can be used to detect faults of the Tow-Thomas filter. If some row is different with other rows, it means that its corresponding fault can be uniquely located. If two or more rows are the same, this means that their corresponding faults cannot be uniquely located. From Table 2, we can see that faults $f_{2}$, $f_{3,} f_{4,} f_{10}, f_{11}, f_{12,}, f_{13}, f_{14}$ can be uniquely located. Other faults cannot be uniquely located. There are three ambiguity sets: $\left\{f_{1}, f_{9}\right\},\left\{f_{6}, f_{8}, f_{16}\right\}$, and $\left\{f_{0}, f_{5}, f_{7}, f_{15}\right\}$. Faults in the same ambiguity sets will be confused each other.

Table 2. Integer-coded faulty-wise dictionary of output voltage

\begin{tabular}{c|cccccccc}
\hline Faults & $t_{1}$ & $t_{2}$ & $t_{3}$ & $t_{4}$ & $t_{5}$ & $t_{6}$ & $t_{7}$ & $t_{8}$ \\
\hline$f_{0}$ & 4 & 4 & 1 & 1 & 4 & 5 & 6 & 6 \\
$f_{1}$ & 3 & 3 & 1 & 1 & 3 & 3 & 4 & 4 \\
$f_{2}$ & 7 & 7 & 1 & 1 & 7 & 7 & 8 & 8 \\
$f_{3}$ & 6 & 6 & 1 & 1 & 6 & 6 & 7 & 7 \\
$f_{4}$ & 2 & 2 & 1 & 1 & 2 & 2 & 3 & 3 \\
$f_{5}$ & 4 & 4 & 1 & 1 & 4 & 5 & 6 & 6 \\
$f_{6}$ & 3 & 3 & 1 & 1 & 3 & 1 & 1 & 1 \\
$f_{7}$ & 4 & 4 & 1 & 1 & 4 & 5 & 6 & 6 \\
$f_{8}$ & 3 & 3 & 1 & 1 & 3 & 1 & 1 & 1 \\
$f_{9}$ & 3 & 3 & 1 & 1 & 3 & 3 & 4 & 4 \\
$f_{10}$ & 5 & 5 & 1 & 1 & 5 & 4 & 4 & 4 \\
$f_{11}$ & 5 & 5 & 1 & 1 & 5 & 5 & 6 & 6 \\
$f_{12}$ & 1 & 1 & 1 & 1 & 1 & 1 & 2 & 2 \\
$f_{13}$ & 4 & 4 & 1 & 1 & 4 & 4 & 5 & 5 \\
$f_{14}$ & 4 & 4 & 1 & 1 & 4 & 3 & 4 & 4 \\
$f_{15}$ & 4 & 4 & 1 & 1 & 4 & 5 & 6 & 6 \\
$f_{16}$ & 3 & 3 & 1 & 1 & 3 & 1 & 1 & 1 \\
\hline
\end{tabular}

\section{Fault features analysis of power supply current}

From Table 2, we can see that these soft faults cannot be uniquely located by using the output voltage only. There are ambiguity sets which contain two or more faults. So, other signals need to be observed. The dynamic power supply current is a new testable signal. Because the CUT need not to be destructed when we test the power supply current. The signal is tested from the power terminal. This is suitable for the fault diagnosis of integrated circuits. Power supply current could be expressed as a function of the branch currents in the circuit. Every fault condition can be considered as a change in some branch currents and it will cause a more or less significant change in the power supply current. In this paper, the dynamic power supply current is used for fault diagnosis of analog circuits. The results were compared with those using the signal of output voltage.

The power supplies for the circuit in Figure 1 are a $12 \mathrm{~V} \mathrm{DC}$ and a $-12 \mathrm{~V} \mathrm{DC}$. The currents passing through the $12 \mathrm{~V} \mathrm{DC}$ and the $-12 \mathrm{~V}$ DC are measured separately under the application of the same stimulus in Section 2. So, two power supply current signals can be acquired from one circuit board. For the Tow-Thomas filter, the currents passing through the $12 \mathrm{~V} \mathrm{DC}$ and the $-12 \mathrm{~V} \mathrm{DC}$ are the same. Therefore, we can use anyone of them to diagnose the circuit. Figure 4 shows the waveform of power supply current when the filter has the faulty type $f_{1}$.

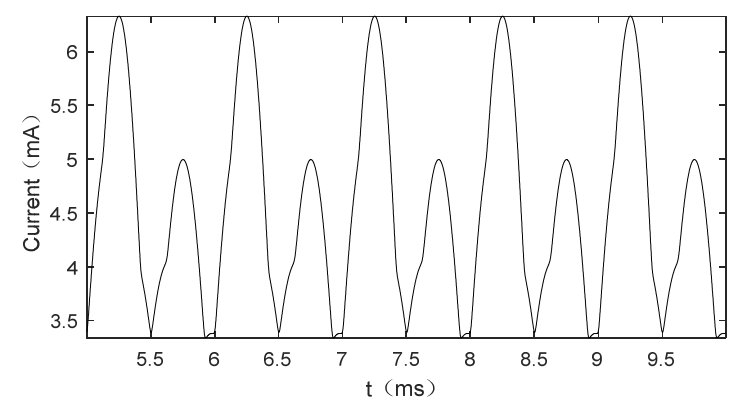

Figure 4. Waveforms of power supply current for the faulty type $f_{1}$

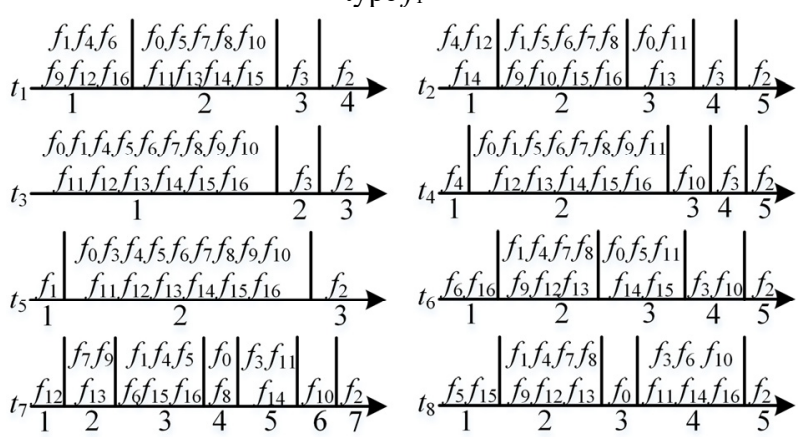

Figure 5. Ambiguity sets for fault features of power supply current

Table 3. Integer-coded faulty-wise dictionary of power supply current

\begin{tabular}{c|cccccccc}
\hline Faults & $t_{1}$ & $t_{2}$ & $t_{3}$ & $t_{4}$ & $t_{5}$ & $t_{6}$ & $t_{7}$ & $t_{8}$ \\
\hline$f_{0}$ & 2 & 3 & 1 & 2 & 2 & 3 & 4 & 3 \\
$f_{1}$ & 1 & 2 & 1 & 2 & 1 & 2 & 3 & 2 \\
$f_{2}$ & 4 & 5 & 3 & 5 & 3 & 5 & 7 & 5 \\
$f_{3}$ & 3 & 4 & 2 & 4 & 2 & 4 & 5 & 4 \\
$f_{4}$ & 1 & 1 & 1 & 1 & 2 & 2 & 3 & 2 \\
$f_{5}$ & 2 & 2 & 1 & 2 & 2 & 3 & 3 & 1 \\
$f_{6}$ & 1 & 2 & 1 & 2 & 2 & 1 & 3 & 4 \\
$f_{7}$ & 2 & 2 & 1 & 2 & 2 & 2 & 2 & 2 \\
$f_{8}$ & 2 & 2 & 1 & 2 & 2 & 2 & 4 & 2 \\
$f_{9}$ & 1 & 2 & 1 & 2 & 2 & 2 & 2 & 2 \\
$f_{10}$ & 2 & 2 & 1 & 3 & 2 & 4 & 6 & 4 \\
$f_{11}$ & 2 & 3 & 1 & 2 & 2 & 3 & 5 & 4
\end{tabular}




\begin{tabular}{l|llllllll}
$f_{12}$ & 1 & 1 & 1 & 2 & 2 & 2 & 1 & 2 \\
$f_{13}$ & 2 & 3 & 1 & 2 & 2 & 2 & 2 & 2 \\
$f_{14}$ & 2 & 1 & 1 & 2 & 2 & 3 & 5 & 4 \\
$f_{15}$ & 2 & 2 & 1 & 2 & 2 & 3 & 3 & 1 \\
$f_{16}$ & 1 & 2 & 1 & 2 & 2 & 1 & 3 & 4
\end{tabular}

In order to diagnose the faults of CUT, we need to extract the fault features of power supply current, too. The same features of time-domain and frequencydomain are selected as those in Section 2. The eight fault features of power supply current and the fault types are used to construct the fault dictionary. The fault features and their ambiguity sets are shown in Figure 5. According these ambiguity sets, the integer-coded faulty-wise dictionary of power supply current are constructed and shown in Table 3. From Figure 5, we can see that the ambiguity sets corresponding to $t_{3}$ are the subset of ambiguity sets corresponding to $t_{4}$. This means that if a fault can be located by $t_{3}$, it can be located by $t_{4}$, too. But if cannot be located by $t_{3}$, it may be located by $t_{4}$. So, we can remove the column corresponding to $t_{3}$.

This simplified fault dictionary of power supply current can be used to detect faults of the Tow-Thomas filter. From Table 3, we can see that the faults can be uniquely located are as follows: $f_{0,}, f_{1,}, f_{2}, f_{3}, f_{4,}, f_{7}, f_{8}, f_{9,}, f_{10}, f_{11}$, $f_{12,} f_{13,} f_{14}$. Four faults cannot be uniquely located. They belong to two ambiguity sets: $\left\{f_{5}, f_{15}\right\}$ and $\left\{f_{6}, f_{16}\right\}$.

\section{Comparing results of two signals}

Fault features of output voltage and power supply current are extracted for fault diagnosis of analog circuits in Section 2 and 3, respectively. The comparing results of these two signals are shown in Table 4. Eight types of faults $f_{2,}, f_{3}, f_{4}, f_{10}, f_{11}, f_{12}, f_{13}, f_{14}$ can be uniquely located by using the output voltage signal. They are $50 \%$ of all the fault types. Thirteen types of faults can be uniquely located if the power supply current signal is used. The proportion is increased to $76.47 \%$. The number of ambiguity sets for output voltage is 3 . The first ambiguity set contains three faults $f_{6}, f_{8}, f_{16}$. But the fault $f_{8}$ can be uniquely located by using the power supply current. This ambiguity set is reduced to $\left\{f_{6}, f_{16}\right\}$. Let we see the second ambiguity set. It contains four faults $f_{0,} f_{5}$, $f_{7,} f_{15}$ when the output voltage is used. But the faults $f_{0,}, f_{7}$ can be uniquely located by using the power supply current. This ambiguity set is reduced to $\left\{f_{5,} f_{15}\right\}$. For the third ambiguity set which contains faults $f_{1}, f_{9}$, it is disappeared by using the power supply current.

Table 4. Comparing results of two signals

\begin{tabular}{c|cc}
\hline Signals & Output Voltage & $\begin{array}{c}\text { Power Supply } \\
\text { Current }\end{array}$ \\
\hline $\begin{array}{c}\text { Number of faults be } \\
\text { uniquely located } \\
\text { Ambiguity Set 1 }\end{array}$ & 8 & 13 \\
Ambiguity Set 2 & $f_{6}, f_{8}, f_{16}$ & $f_{6}, f_{16}$ \\
Ambiguity Set 3 & $f_{0}, f_{5}, f_{7}, f_{15}$ & $f_{5}, f_{15}$ \\
\hline
\end{tabular}

\section{Conclusions}

Fault diagnosis of analog circuits is the bottleneck of electronic circuits at present. The soft faults are more difficult to diagnose than the hard faults because the soft faults are caused by the deviations of component parameters. The fault features of tested signal should be analyzed properly. Because they are critical for the fault diagnosis of analog circuits. In this paper, the features of both time-domain and frequency-domain are analyzed and used to diagnose analog circuits. Firstly, the fault features of output voltage signal were analyzed and used to diagnosis the Tow-Thomas filter. The results show that eight type of faults can be uniquely located by using the output voltage only. Other nine faults are divided into three ambiguity sets and cannot be uniquely located. So, we need to find other accessible signal. The dynamic power supply current was acquired and its fault features were analyzed. These fault features were used to diagnose the soft faults of Tow-Thomas filter, too. The fault diagnose results were compared with those using the output voltage. The results show that the number of ambiguity sets is decreased to 2 and the number of faults can be uniquely located is increased to 13 if the dynamic power supply current is used. And the dynamic power supply current is an accessible signal, because the CUT need not to be destructed when we test this signal. These indicate that the dynamic power supply current is suitable for fault diagnosis of analog circuits.

\section{Acknowledgment}

The authors acknowledge to the National Natural Science Foundation of China for financial support Project 51509255.

\section{References}

1. M. Aminian, F. Aminian, A modular faultdiagnostic system for analog electronic circuits using neural networks with wavelet transform as a preprocessor, IEEE Transactions on Instrumentation and Measure-ment, 56, 5, pp. 1546-1554 (2007).

2. M. Khanlari, M. Ehsanian, An improved KFCM clustering method used for multiple fault diagnosis of analog circuits, Circuits Syst Signal Process, 36, pp. 3491-3513 (2017).

3. X. Gan, W. Gao, Z. Dai, W. Liu, Research on WNN soft fault diagnosis for analog circuit based on adaptive UKF algorithm, Applied Soft Computing, 50, pp. 252-259 (2017).

4. P. Wang, S. Yang, A new diagnosis approach for handling tolerance in analog and mixed-signal circuits by using fuzzy math, IEEE Transactions on Instrumentation and Measurement, 56, 5, pp. 21182127 (2009).

5. D. K. Papakostas, A. A. Hatzopoulos, Improved analogue fault coverage estimation using probabilistic analysis, International Journal of Circuit Theory and Applications, 38, 5, pp. 503-514 (2010). 
6. V. C. Prasad, N. S. C. Babu, Selection of test nodes for analog fault diagnosis in dictionary approach, IEEE Transactions on Instrumentation and Measurement, 49, 6, pp. 1289-1297 (2000).

7. W. Hochwald, J. D. Bastian, A dc approach for analog fault dictionary determination, IEEE Trans. Circuits Syst. I, CAS-26, pp. 523-529 (1979).

8. C. Yang, S. tian, B. Long, Application of heuristic graph search to test-point selection for analog fault dictionary techniques, IEEE Transactions on Instrumentation and Measurement, 58, 7, pp. 2145 2158 (2003). 